DOI: https://doi.org/10.11144/Javeriana.upsy.17-2.iudt

\title{
Implicación de usuarios diagnosticados de Trastorno de Ansiedad Generalizada en la elaboración de una guía de práctica clínica*
}

\section{Involvement of Users Diagnosed with Generalized Anxiety Disorder in the Development of a Clinical Practice Guideline}

Recepción: 11 Marzo 2016 | Aprobación: 10 Julio 2017

\author{
Gisela Amor Mercado \\ Hospital Regional Universitario de Málaga, España \\ Amanda Vega NúÑ̃Z \\ Hospital Regional Universitario de Málaga, España \\ Amelia Villena Jimena \\ Hospital Regional Universitario de Málaga, España \\ Clara Gómez Ocaña ${ }^{\mathrm{a}}$ \\ Hospital Regional Universitario de Málaga, España \\ ORCID: http://orcid.org/0000-0002-7447-3666 \\ José Miguel Morales-Asencio \\ Universidad de Málaga, España \\ María Magdalena Hurtado Lara \\ Hospital Regional Universitario de Málaga, España
}

\footnotetext{
a Autora de correspondencia. Correo electrónico: clara.gomez321@gmail.com
}

Para citar este artículo: Amor Mercado, G., Vega Núñez, A., Villena Jimena, A., Gómez Ocaña, C., Morales-Asencio, J. M., \& Hurtado Lara, M. M. (2018). Implicación de usuarios diagnosticados de Trastorno de Ansiedad Generalizada en la elaboración de una guía de práctica clínica. Universitas Psychologica, 17(2), 1-10. https://

doi.org/10.11144/Javeriana.upsy.17-2.iudt

\section{RESUMEN}

En la actualidad hay un interés creciente en implicar a los usuarios de los servicios sanitarios en la elaboración de guías de práctica clínica, especialmente en los problemas de salud de mayor complejidad o frecuencia, como es el Trastorno de Ansiedad Generalizada (TAG). El objetivo del presente artículo es dar a conocer la novedosa metodología cualitativa utilizada para maximizar el impacto del punto de vista de un grupo de usuarios con TAG en la elaboración de una Guía de práctica clínica (GPC) sobre dicho trastorno. Para ello, se realizaron grupos focales y, a partir del análisis de contenido, se vincularon los testimonios de los usuarios con las recomendaciones basadas en la evidencia, situando ambas fuentes de información al mismo nivel de relevancia.

\section{Palabras clave}

guía de práctica clínica; trastorno de ansiedad generalizada; participación del paciente; investigación cualitativa.

\begin{abstract}
Currently, there is an increasing interest in involving the health service users in the development of Clinical practice guidelines (CPG), specially in the more complex and frequent health problems, as the Generalized Anxiety Disorder (GAD). The purpose of this article is to communicate the novel qualitative methodology that has been used to maximize the impact of the perspective of a group of users with GAD in the development of a CPG about it. To that end, focal groups were performed and, from the content analysis, the users testimonials were linked to the evidence-based
\end{abstract}


recommendations of the CPG based on the same topic, in order to situate both sources of information at the same level of relevance.

Keywords

practice guideline; generalized anxiety disorder; patient participation; qualitative research.

Las Guías de práctica clínica (GPC) permiten dar a conocer las estrategias y la evidencia científica relacionada con el abordaje de un determinado problema de salud. Hasta hace unos años, han sido los profesionales los únicos implicados en la elaboración de las GPC. Sin embargo, en la actualidad hay un interés creciente en que los usuarios diagnosticados del problema de salud tengan una mayor implicación en el proceso de elaboración (Díaz del Campo, Gracia, Blasco \& Andradas, 2011). Mejorar la aceptación y el seguimiento de la guía es uno de los objetivos que se persiguen con esta implicación. No obstante, no hay consenso acerca de la manera más adecuada de llevar a cabo dicha implicación de los usuarios (Grupo de trabajo de implicación de pacientes en el desarrollo de GPC, 2013; Lincoln, Lynham,\& Guba, 2011).

El trastorno de ansiedad generalizada (TAG) es el segundo trastorno mental más frecuente después de la depresión y, dentro de los trastornos ansiosos, el más frecuente en atención primaria (Üstün \& Sartorius, 1995). El curso crónico y fluctuante de la sintomatología del TAG, el hecho de que la mayoría de las personas con TAG no busquen ayuda sanitaria (Wittchen $\&$ Jacobi, 2005) y el bajo reconocimiento del trastorno en atención primaria (Roy-Byrne \& Wagner, 2004; Wittchen et al., 2002; Wittchen \& Jacobi, 2005) dificultan la realización de un diagnóstico acertado y, por consiguiente, la elección del tratamiento adecuado (McManus, Meltzer, Brugha, Bebbington, \& Jenkins, 2009), lo que finalmente da lugar a la cronificación y a una mayor utilización de los recursos sanitarios, que se asocian con altos costes médicos y sociales (Simon, Ormel, von Korff, \& Barlow, 1995). Estas peculiaridades del TAG hacen que conocer la opinión de los usuarios respecto al trastorno y a la atención sanitaria pueda resultar de gran relevancia.

Desde la Unidad de Gestión Clínica (UGC) de salud mental del Hospital Regional de Málaga se ha desarrollado una GPC para el tratamiento del TAG (García-Herrera Pérez-Bryan, Hurtado Lara, Nogueras Morillas, Bordallo Aragón,\& Morales Asencio, 2015), basada en el modelo de atención por pasos, con el objeto de mejorar la detección, el establecimiento del manejo en el nivel adecuado, el desarrollo de criterios para el tratamiento, el seguimiento y la derivación de este trastorno. Esta GPC se ha elaborado siguiendo el método estructurado de adaptación de GPC del ADAPTE Working Group, y está basada en la GPC para el TAG del National Institute for Mental Health and Care Excellence (NICE, 2011), al ser la guía que obtuvo mejor calificación de las siete analizadas con el instrumento AGREE II (The AGREE Collaboration, 2001). Esta guía (NICE, 2011) incluye, en su capítulo 4, relatos de personas con TAG y sus familiares sobre la vivencia del trastorno y la atención sanitaria recibida. Siguiendo esta línea, y teniendo en cuenta la relevancia que la opinión de los usuarios está adquiriendo en los últimos años, en esta GPC (García-Herrera Pérez-Bryan et al., 2015) no solo se elaboró una sección sobre la experiencia de los usuarios, sino que se ideó una metodología que permitiera situar los testimonios de los usuarios al mismo nivel de relevancia que las recomendaciones basadas en la evidencia.

El objetivo del presente artículo es dar a conocer la metodología utilizada para ello. Por primera vez en una GPC, se realiza una vinculación de los testimonios de los usuarios con las recomendaciones basadas en la evidencia (García-Herrera Pérez-Bryan et al., 2015). Adicionalmente, se pretende explorar los resultados más representativos de este proceso.

\section{Método}

La metodología seguida se puede dividir en dos fases. La primera de ellas consistió en investigación cualitativa, basada en grupos 
focales, acerca de los testimonios y experiencias de los usuarios en relación al proceso de ansiedad. En una segunda fase, se realizó una vinculación de los testimonios de los informantes con la formulación de las recomendaciones basadas en la evidencia. Con esta segunda fase se pretende mejorar la adherencia a las recomendaciones, especialmente en el caso de aquellas que coinciden con los valores y preferencias de los usuarios.

\section{Primera fase: Estudio cualitativo}

El estudio cualitativo fue llevado a cabo en la UGC de salud mental del Hospital Regional Universitario de Málaga, concretamente en la Unidad de salud mental Comunitaria MálagaCentro. Este dispositivo atiende habitualmente a una población de 165.000 habitantes, y ofrece atención psiquiátrica y psicológica a personas con distintos trastornos mentales, así como terapias grupales dirigidas específicamente a distintos perfiles psicopatológicos. Esta unidad atiende aproximadamente a unos 170 usuarios con diagnóstico de TAG al año.

\section{Sujetos del estudio}

La selección de los usuarios se realizó teniendo en cuenta los criterios recogidos en la Tabla 1.

\section{Tabla 1}

\section{Criterios de selección}

\begin{tabular}{ll}
\multicolumn{1}{c}{ Criterios de inclusión } & \multicolumn{1}{c}{ Criterios de exclusión } \\
\hline Diagnóstico de TAG, realizado por & Minoría de edad. \\
psicólogos clínicos o psiquiatras, de & Diagnóstico de trastorno mental grave \\
acuerdo al manual diagnóstico y & (trastornos psicóticos o bipolares) o una \\
estadístico de los trastornos mentales & adicción comórbida (alcohol o alguna \\
DSM-5. & droga ilegal). \\
Al menos dos citas en salud mental, & Retraso mental, coeficiente intelectual \\
además de las consultas en atención & límite o deterioro funcional importante. \\
primaria. & Abandono del seguimiento sanitario sin \\
& recibir el alta por parte de un facultativo. \\
& Situación social marginal, o no saber leer. \\
\hline
\end{tabular}

Mediante muestreo intencional, y teniendo en cuenta los criterios mencionados, se revisó el listado completo de usuarios a los que se les diagnosticó TAG. Una vez elegidos los pacientes, fueron contactados telefónicamente todos los pre-seleccionados, para explicarles los objetivos de la investigación y solicitar su participación.
Los usuarios que aceptaron participar en el estudio fueron citados el día del grupo con cierta antelación para que pudieran leer la hoja de información al paciente y hacer las preguntas que estimasen oportunas antes de firmar el consentimiento informado. No se utilizaron otros criterios de segmentación de los grupos.

El tamaño muestral estuvo sometido al principio de saturación de la información durante el proceso de recogida de datos y análisis.

\section{Recolección de los datos}

La técnica empleada para la obtención de los datos fue la entrevista grupal mediante grupos focales. Las entrevistas fueron de tipo semi-estructuradas, apoyadas en la ayuda de un guion. Para la construcción del este se tuvieron en cuenta aspectos identificados en la literatura, se realizaron entrevistas a expertos y finalmente se optó por adaptar las preguntas de la encuesta llevada a cabo por el equipo de trabajo desarrollador de la guía NICE para el TAG (2011). Finalmente, las temáticas abordadas fueron el impacto del TAG en la vida cotidiana y las relaciones interpersonales, la valoración de los profesionales implicados (atención primaria y salud mental) y del proceso asistencial,los tipos de abordajes ofertados en el sistema sanitario público - psicológicos y farmacológicos - y su utilidad percibida, y los recursos personales de afrontamiento de la ansiedad. Las preguntas fueron de carácter abierto, y la entrevista se llevó a cabo en un estilo flexible, de manera que pudieran emerger temas no propuestos inicialmente. Las entrevistas tuvieron una duración de entre 90 y 120 minutos.

\section{Análisis de los datos}

Las entrevistas fueron grabadas en audio y transcritas literalmente. Se realizó un análisis de contenido de acuerdo con los principios de Taylor y Bodgan (1998). Se identificaron temas emergentes tras varias lecturas sucesivas de las entrevistas. Posteriormente, las citas significativas fueron codificadas por un 
investigador. Estos códigos fueron triangulados por medio de una revisión por otros investigadores del equipo. Las divergencias en los códigos propuestos se discutieron entre los investigadores hasta que se obtuvo un acuerdo final. Los códigos se agruparon en categorías y subcategorías y fueron analizados teniendo en cuenta la potencial influencia derivada de los investigadores. Todos los análisis se realizaron con el software de datos cualitativos ATLAS Ti 7.

\section{Credibilidad y validez}

Para garantizar la credibilidad y validez de los resultados, se tuvieron en cuenta los criterios de Lincoln et al. (2011): credibilidad, transferibilidad, consistencia y confirmabilidad. Para garantizar la credibilidad en el proceso de análisis, se procedió a la triangulación de códigos y categorías. La transferibilidad se afianzó mediante una recogida exhaustiva de los datos, la descripción pormenorizada de los participantes, y la cantidad de información recogida en cada grupo, de manera que quedase garantizada la disponibilidad de información suficiente. El criterio de consistencia y replicabilidad de los datos se siguió a través de un proceso detallado y documentado de la estrategia de análisis, así como del contexto en el que tuvo lugar la recogida de datos. Desde el punto de vista de la confirmabilidad y la reflexividad antes del inicio del estudio, los investigadores llevaron a cabo un análisis de ideas preconcebidas y expectativas propias con respecto a los resultados del estudio para poder comparar posteriormente en qué medida pudieron influir a lo largo del proceso. Además, se utilizó un moderador/ entrevistador neutro (no perteneciente al equipo de investigación) y con experiencia en entrevistas cualitativas.

\section{Aspectos éticos}

El estudio fue autorizado por el Comité de Ética de la Investigación Provincial de Málaga. Todos los participantes fueron informados por escrito y verbalmente con antelación a la solicitud de su consentimiento para participar en el estudio. En todo momento se mantuvieron los principios de buena práctica y los preceptos de la Declaración de Helsinki y sus revisiones posteriores. Los datos fueron tratados de forma confidencial y anonimizados para su tratamiento durante el análisis.

Segunda fase: Proceso de vinculación de los resultados a las recomendaciones de la GPC

Una vez realizado el análisis de contenido, y con el objetivo de mejorar la predisposición a la adherencia del lector profesional a las recomendaciones, se realizó un emparejamiento de los testimonios de los informantes y de los resultados más relevantes del estudio con las recomendaciones basadas en la evidencia. El procedimiento seguido comenzó con la lectura detallada de los relatos de los usuarios, proceso mediante el cual se identificaron los resultados con mayor relevancia clínica y se procedió a identificar en la lista de recomendaciones posibles vínculos relacionales entre los testimonios y cada recomendación. Este proceso de vinculación fue llevado a cabo por los miembros del grupo desarrollador de la guía que llevaron a cabo el análisis cualitativo. Los emparejamientos iniciales se distribuyeron al resto de los miembros de dicho grupo para que contrastaran y contextualizaran dichas asignaciones, en donde se resolvieron las discrepancias mediante consenso.

\section{Resultados}

\section{Características de los participantes}

La muestra finalmente obtenida fue de 10 participantes, que aceptaron formar parte de la investigación y pudieron acudir de hecho a la cita prevista. En la Tabla 2 se recogen las principales características de la muestra. 
Tabla 2

Características de los participantes

\begin{tabular}{ll}
\hline \multicolumn{1}{c}{ Características } & \multicolumn{1}{c}{ Descriptivos } \\
\hline Edad (en años) & Media: 50.6 \\
& Rango: $24-66$ \\
\hline Género & Mujeres: 8 \\
& Hombres: 2 \\
\hline Estado civil & Soltero: 2 \\
& Con pareja: 7 \\
& Separado: 1 \\
\hline Tiempo de seguimiento en & Media: 38.2 \\
salud mental (en meses) & Rango: $4-120$ \\
\hline
\end{tabular}

Todos los informantes, excepto dos, tienen diagnósticos comórbidos con otros trastornos mentales comunes (depresión y/u otros trastornos de ansiedad). Se realizaron dos grupos focales, con cuatro y seis participantes respectivamente.

Vinculación de los testimonios con las recomendaciones

Los resultados obtenidos tras el análisis cualitativo del testimonio de los participantes en los grupos focales fueron agrupados en cuatro grandes áreas temáticas: inicio del trastorno y síntomas, la vida cotidiana (interferencia y apoyos), el afrontamiento del trastorno y la relación con el sistema de salud.

Una vez realizado dicho análisis, los resultados más relevantes para la práctica clínica, rescatados de los testimonios de los informantes, se vincularon a las recomendaciones basadas en la evidencia de la GPC con las que estuvieron relacionadas. Así, junto a algunas recomendaciones, se añadió un resumen de la opinión de los usuarios al respecto, extraído de la investigación cualitativa y esto, a su vez, se acompañó de un extracto literal de los comentarios de los usuarios, a modo de ejemplo. Para mayor contextualización, se indica sexo y edad del informante.

Con respecto a los resultados hallados, en primer lugar destaca la queja manifestada por la mayoría de los participantes por la falta de información que se les proporciona acerca del trastorno, no solo en los servicios de urgencias o atención primaria, sino incluso en los Servicios de salud mental, a pesar de que es una recomendación básica de la GPC. Por ello, en la recomendación 6.8 de esta GPC (García-Herrera Pérez-Bryan et al., 2015), no solo se aconseja a los profesionales proporcionar a los usuarios "educación sobre la naturaleza del TAG y las opciones de tratamiento", sino que se deja constancia de la opinión de los usuarios al respecto, ya que recibir este tipo de información no solo es el resultado de seguir una recomendación, sino que también es una necesidad percibida por los usuarios.

Por otra parte, muchos informantes refirieron haberse beneficiado considerablemente en los grupos focales de que algún facultativo de salud mental informara a sus familiares sobre la naturaleza del trastorno, al percibir escasa comprensión por parte de los mismos, lo que supuso una mejora importante del apoyo familiar y social percibido. Así, en la recomendación 7.3 de la GPC se deja constancia de ello, reforzando así la indicación de implicar a los familiares y cuidadores proporcionando información sobre el TAG y atención a los mismos.

En cuanto al diagnóstico, a pesar de que identificarlo y comunicarlo es una de las recomendaciones basadas en la evidencia (recomendación 7.6), ha resultado llamativo apreciar que ninguno de los participantes que acudieron a los grupos focales mencionó en ningún momento su diagnóstico exacto (TAG), aunque sí referían sintomatología ansiosa. Esto sugiere que dicha recomendación no está siendo aplicada de forma generalizada por los profesionales sanitarios, en cuyo caso se trataría de un área de mejora.

En relación a la recomendación de ofrecer una o más intervenciones psicológicas de baja intensidad en función de la preferencia de la persona, si los síntomas no han mejorado después de la psicoeducación y el seguimiento activo (recomendación 10.1), el estudio cualitativo pone de manifiesto que el tratamiento de primera elección fue el farmacológico en la mayoría de los casos, aunque bien es cierto que muchos de ellos fueron derivados a tratamientos psicológicos grupales en diferentes momentos del 
seguimiento, siendo esta última opción valorada de forma muy positiva por todos los informantes. Por otra parte, ninguno de ellos mencionó haber recibido material de autoayuda.

En la misma línea, en la GPC se recomienda basar la elección de tratamiento en la preferencia de las personas, ya que no hay evidencia de la superioridad del tratamiento psicológico frente al farmacológico. El estudio cualitativo confirma que la mayor parte de los usuarios manifiestan una clara preferencia por el tratamiento psicológico y que, además, perciben una escasa accesibilidad a este tipo de abordaje en la sanidad pública, en donde muchos de ellos llegan a plantearse acudir a terapia psicológica en el ámbito privado.

Finalmente, vinculada a las recomendaciones 11.5 y 11.6, referidas a intervenciones psicológicas para el tratamiento del TAG (Terapia Cognitivo Conductual y relajación), y a cómo estas deben ser aplicadas, se deja constancia de la queja manifestada por muchos usuarios con respecto a la frecuencia de las citas en salud mental, que consideran demasiado espaciadas. Lo mismo ocurre con las recomendaciones 12.9 y 12.11 , que aconsejanuna monitorización y revisión frecuente del tratamiento farmacológico, pero que los pacientes perciben como insuficiente, ya que incluso refieren meses de espera entre consultas, lo que asocian a una merma en su evolución.

El Anexo 1 recoge las recomendaciones más representativas de la GPC a las que se les vinculó el testimonio de los usuarios.

\section{Discusión}

El objetivo de este artículo es dar a conocer la metodología utilizada en la implicación de usuarios diagnosticados de TAG en el desarrollo de una GPC sobre el mismo. Tras realizar un estudio cualitativo con usuarios de nuestro contexto, se elaboró un capítulo específico sobre la experiencia del usuario y, además, se realizó una vinculación de las recomendaciones basadas en la evidencia recogida en la GPC
(García-Herrera Pérez-Bryan et al., 2015) con las preferencias, necesidades y demandas de este grupo de usuarios. También se han expuesto las principales conclusiones extraídas del análisis cualitativo.

Este estudio ha sido motivado por la especial importancia que está cobrando en los últimos años la "voz" de los usuarios, pues se considera que la participación de los mismos en el proceso de elaboración de una GPC puede garantizar su aceptación y el seguimiento de las recomendaciones, así como permitir un conocimiento más profundo del problema clínico (Grupo de trabajo de implicación de pacientes en el desarrollo de GPC, 2013; Harding, Pettinari, Brown, Hayward,\& Taylor, 2011). Aunque cada vez más se llevan a cabo experiencias similares, como Guidelines International Network, Scottish Intercollegiate Guidelines Network y National Institute for Health and Clinical Excellence, este trabajo presenta la novedad de vincular las opiniones de los usuarios con las recomendaciones basadas en la evidencia, situándolas en un mismo nivel de relevancia.

La metodología usada en esta GPC (GarcíaHerrera Pérez-Bryan et al., 2015) ha permitido identificar áreas de mejora en la atención a este tipo de pacientes, así como conocer la satisfacción con la respuesta sanitaria recibida. De este modo, se ha puesto de manifiesto que los usuarios demandan mayor información y psicoeducación sobre su trastorno, y una vez este se ha identificado, mayor implicación de la familia en el proceso de atención sanitaria, de modo que esta también comprenda la naturaleza del mismo; consultas más frecuentes con los facultativos de salud mental, lo que pone de manifiesto que no se cumplen los criterios temporales de intervención recomendados por la GPC, ni en el tratamiento farmacológico ni en el psicológico, y que prefieren el abordaje psicoterapéutico frente al tratamiento farmacológico.

Con la vinculación de estos testimonios a las recomendaciones, se deja constancia de estas percepciones y demandas de los usuarios, de modo que refuerzan la importancia de aplicación 
de las mismas en la práctica clínica en la medida que sea posible. Así, la información sobre la evidencia empírica y sobre la opinión de los usuarios se presenta fácilmente accesible al profesional que consulte la GPC para orientarse en el proceso de toma de decisiones, lo cual repercutirá en un mejor acercamiento al problema concreto y una atención más centrada en el usuario.

Estas vinculaciones son presentadas en la GPC (García-Herrera Pérez-Bryan et al., 2015), preludiadas por una revisión bibliográfica sobre investigaciones cualitativas en pacientes con trastornos de ansiedad generalizada.

Muchos autores resaltan la importancia de estudiar si la implicación de los usuarios en las guías tiene los efectos esperados sobre su aceptación y seguimiento de las recomendaciones (Harding et al., 2011; Lincoln et al., 2011; van Wersch \& Eccles, 2001). Otras de las ventajas que puede presentar y que ha sido menos señalada, hace referencia al efecto que puede tener en los participantes. Así, esta implicación fomenta la toma de decisiones, disminuye el paternalismo y, consecuentemente, podría fomentar el empoderamiento de los usuarios, así como debilitar el estigma asociado a los trastornos mentales, por lo que tendría también, una valor terapéutico añadido (Deegan $\&$ Drake, 2006; Harding et al., 2011).

\section{Conclusiones}

El objetivo de este artículo es dar a conocer la novedad de vincular el testimonio de usuarios diagnosticados de TAG con las recomendaciones de la GPC sobre el TAG, realizada por la UGC de salud mental del Hospital Regional de Málaga. Esto ha surgido del mayor reconocimiento que está teniendo en los últimos años la necesidad de "dar voz" a los usuarios de los servicios sanitarios en su propio proceso psicopatológico.

Para ello, la metodología privilegiada es la perspectiva cualitativa, que proporciona información útil para la práctica asistencial, y resuelve preguntas que no pueden ser abordadas desde la epidemiología. En el ámbito de la salud mental, la investigación cualitativa resulta de especial relevancia ya que permite profundizar en la naturaleza de los trastornos y del proceso de recuperación mediante el acercamiento a las experiencias que tienen las personas que los padecen, así como los de su alrededor.

Este tipo de innovaciones en la elaboración de GPC permiten la implicación efectiva de los usuarios en este proceso, lo cual puede constituir una garantía de que el texto trata aquellos aspectos que son importantes para ellos y que sus puntos de vista están siendo adecuadamente recogidos.

\section{Referencias}

Deegan, P.E.,\& Drake, R.E. (2006). Shared decision making and medication management in the recovery process. Psychiatric Services, 57(11), 1636-1639.

Díaz del Campo, P., Gracia, J., Blasco, J., \& Andrada, E. (2011). A strategy for patient involvement in clinical practice guidelines: methodological approaches. BMJ Quality $\mathbb{E}$ Safety, 20 (9), 779-784. https://doi.org/10.1 136/bmjqs.2010.049031

García-Herrera Pérez-Bryan, J. M., Hurtado Lara, M. M., Nogueras Morillas, V., Bordallo Aragón, A.,\& Morales Asencio, J. M. (2015). Guía de práctica clínica para el Tratamiento del Trastorno de Ansiedad Generalizada. Basada en el modelo de atención por pasos en Atención Primaria y en Salud Mental. Guía completa. Málaga: Hospital Regional de Málaga.

Grupo de trabajo de implicación de pacientes en el desarrollo de GPC. (2013). Implicación de Pacientes en el Desarrollo de Guías de Práctica Clínica: Manual Metodológico. Aragón: Ministerio de Sanidad, Servicios Sociales e Igualdad.

Harding, E., Johnson, C. J., Brown, D., Hayward, M., \& Taylor, C. (2011). Service user involvement in clinical guideline development and implementation: Learning from mental health service users in the UK. International 
Review of Psychiatry, 23(4), 352-357. https:/ /doi.org/10.3109/09540261.2011.606802

Lincoln, Y., Lynham, S.,\& Guba, E. (2011). Paradigmatic controversies, contradictions, and emerging confluences, revisited. En N.Denzin \& Y.Lincoln (Eds.), The SAGE handbook of qualitative research (pp. 97-128). Thousand Oaks: SAGE publications.

McManus, S., Meltzer, H., Brugha, T., Bebbington, P., \& Jenkins, R. (2009). Adult Psychiatric Morbidity in England, 2007: Results of a Household Survey. Leeds: The NHS Information Centre for Health and Social Care.

National Institute for Mental Health and Care Excellence. (2011). Generalised anxiety disorder and panic disorder (with or without agoraphobia) in adults: management in primary, secondary and community care (full NICE guideline). Leicester: The British Psychological Society.

Roy-Byrne, P. P., \& Wagner, A. (2004). Primary care perspectives on generalized anxiety disorder. Journal of Clinical Psychiatry, 65 (supl. 13), 20-26.

Simon, G., Ormel, J., VonKorff, M.,\& Barlow, W. (1995). Health care costs associated with depressive and anxiety disorders in primary care. American Journal of Psychiatry, 152(3), 352-357.

Taylor, S.,\& Bodgan, R. (1998). Introduction to qualitative research methods: a guidebook and resource. Nueva York: Wiley.

The AGREE Collaboration. (2001). AGREE Instrument Spanish version. Recuperado de http://www.agreecollaboration.org

Üstün, T. B.,\& Sartorius, N. (1995). Mental illness in general health care. An international study. Chichester: John Wiley and Sons.

vanWersch, A.,\& Eccles, M. (2001). Involvement of consumers in the development of evidence based clinical guidelines: Practical experiences from the north of England evidence based guideline development programme. Quality in Health Care, 10(1), 10-16.

Wittchen, H. U.,\& Jacobi, F. (2005).Size and burden of mental disorders in Europe: a critical review and appraisal of 27 studies. European Neuropsychopharmacology, 15(4), 357-376.

Wittchen, H. U., Kessler, R. C., Beesdo, K., Krause, P., Höfler, M.,\& Hoyer, J. (2002). Generalized anxiety and depression in primary care: prevalence, recognition, and management. Journal of Clinical Psychiatry, 63 (supl. 8), 24-34.

\section{Anexo \\ Ejemplos de los emparejamientos finales testimonios-recomendaciones \\ INFORMACIÓN SOBRE LA NATURALEZA DEL TRASTORNO}

\author{
6.8. Después de la evaluación y el \\ diagnóstico del TAG:
}

Proporcione a la persona educación sobre la naturaleza del TAG y las opciones de tratamiento, incluyendo información escrita acerca de este trastorno de ansiedad. Realice un seguimiento activo de los síntomas de la persona y de su funcionamiento. La educación $\mathrm{y}$ el seguimiento activo pueden mejorar presentaciones leves y evitar la necesidad de nuevas intervenciones.

Opinión de los usuarios:

Una parte considerable de las personas con TAG que han participado en los grupos focales se quejan de que se les proporciona poca información acerca de su trastorno, incluso cuando se les deriva a salud mental.

Testimonio de los usuarios:

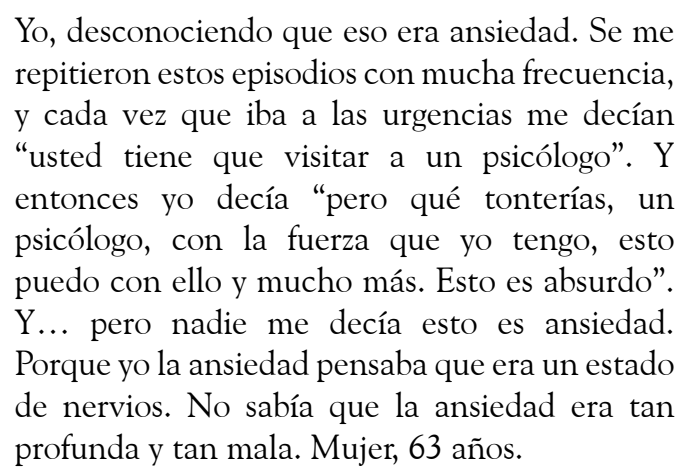

Yo, desconociendo que eso era ansiedad. Se me repitieron estos episodios con mucha frecuencia, y cada vez que iba a las urgencias me decían "usted tiene que visitar a un psicólogo". Y entonces yo decía "pero qué tonterías, un psicólogo, con la fuerza que yo tengo, esto puedo con ello y mucho más. Esto es absurdo". Y... pero nadie me decía esto es ansiedad. Porque yo la ansiedad pensaba que era un estado profunda y tan mala. Mujer, 63 años. 


\section{IMPLICACIÓN DE LA FAMILIA}

7.3. Cuando las familias y los cuidadores están involucrados en el apoyo a una persona con TAG, considere:

Ofrecer una evaluación al cuidador de su tarea de cuidados y de sus necesidades de salud mental y física.

Proporcionar información sobre grupos de apoyo para la familia o cuidadores y organizaciones de voluntarios locales y ayude a las familias o a los cuidadores a acceder a estos.

Negociar entre la persona y su familia o cuidadores la confidencialidad y el intercambio de información.

Proporcionar información escrita y verbal sobre el TAG y su manejo, incluyendo cómo pueden apoyar a la persona.

Proporcionar los números de contacto y la información acerca de qué hacer y a quién contactar en caso de crisis.

Opinión de los usuarios:

Algunos pacientes refieren haber encontrado muy beneficioso que el psiquiatra o psicólogo informe sobre la naturaleza del trastorno a sus familiares escépticos.

Testimonio de los usuarios:

Mi hermano se cree que a mí me encanta ir al médico y me encanta todo eso. Y mi novio, al principio, un poco más de lo mismo que mi hermano, que todo estaba en mi cabeza, que yo me lo imaginaba, y a raíz de que mi suegra, al trabajar con un psiquiatra, un día le dijo "le pasa tal, tal, tal", y él dijo "que pase ella, y que pase el otro". Como que él [el psiquiatra] le explicó a mi novio todo lo que me iba a pasar, como para que fuera inventado por mí, y dijo "vamos a ver, esto y esto, y cuando le pase, tú tienes que actuar de tal manera”. Mujer, 24 años.

\section{DIAGNÓSTICO}

7.6. Identifique y comunique el diagnóstico de TAG tan pronto como sea posible para ayudar a la persona a comprender el trastorno mental y a comenzar un tratamiento efectivo con prontitud.
Opinión de los de los usuarios:

Los participantes en los grupos focales refirieron tener ansiedad, si bien ninguno de ellos mencionó su diagnóstico exacto (TAG).

\section{INTERVENCIONES PSICOLÓGICAS DE BAJA INTENSIDAD}

10.1.Para las personas con TAG cuyos síntomas no han mejorado después de la educación y el seguimiento activo en el paso 1 , ofrezca una o más de las intervenciones psicológicas de baja intensidad de la tabla de abajo, guiado por la preferencia de la persona:

Autoayuda individual no dirigida

Autoayuda individual dirigida

Grupo psicoeducativo

Opinión de los usuarios:

A la mayoría de las personas con TAG que participaron en los grupos focales se les prescribió tratamiento farmacológico como primera opción, muchos de ellos también fueron derivados a tratamiento psicológico grupal en diferentes momentos de la atención sanitaria, y ninguno mencionó que se les proporcionara material de autoayuda. La opinión sobre la utilidad de los grupos de terapia fue unánimemente positiva, y sirvió, incluso, para generar redes de apoyo social.

Testimonios de los usuarios:

Una usuaria, de 63 años, describe que en su primera consulta por una crisis de ansiedad: "Me dieron ansiolíticos y me quedé en cama ese día, pero ya al día siguiente, el día veintisiete, fui a trabajar y seguía con mi mismo ritmo de trabajo" Mujer, 63 años.

"Nos ayudaba de ese tipo de reuniones que tú crees que te pasa lo mayor, y cuando tú escuchas al que está a tu lado, dices 'iy de qué me quejo yo?'. No es tanto el problema tuyo, es mucho más el de quien tienes al lado". Varón, 68 años.

Yo estuve primero, aquí, en un grupo de ansiedad. No, primero fue de agorafobia y me vino súper bien. Ya por lo menos sabía, me explicaron todo como era. Los demás compañeros, pues, igual. Locos por que llegara el jueves, que era el jueves y, luego, hará como dos o tres años, estuve en otro grupo que era de 
ansiedad y también súper bien. De hecho, con los compañeros de este último grupo, seguimos teniendo contacto y nos tenemos puestos "los terapéuticos" y súper bien, muy contentos. Mujer, 39 años.

\section{PREFERENCIA POR LAS INTERVENCIONES PSICOLÓGICAS}

\begin{abstract}
11.3. Base la elección del tratamiento en las preferencias de la persona, ya que no hay evidencia de que cualquiera de los dos tipos de tratamiento (intervención psicológica de alta intensidad individual o el tratamiento farmacológico) sea mejor.
\end{abstract}

Opinión de los usuarios:

Una parte considerable de las personas con TAG que participaron en los grupos focales manifestaron una clara preferencia por el tratamiento psicológico, en donde en algún caso hasta llegaron a negarse a tomar medicación, pero se quejan de la poca accesibilidad a este tipo de abordaje en la sanidad pública.

Testimonio de los usuarios:

Pero eso, yo tengo contra eso, con la Seguridad Social, yo no estoy de acuerdo porque nos mandan siempre al psiquiatra y medicina, y no nos mandan... a mí no me ha visto ningún psicólogo en la Seguridad Social en todo el tiempo que yo llevo con los trastornos del sueño. Yo creo que sería mejor psicólogo y terapia. Mujer, 60 años.

“(...) porque yo he pensado sacarme un seguro particular para ir a un psicólogo. (...) Ya te digo, porque me niego a tomar el medicamento. (...) porque para qué voy a tomar los medicamentos si es para nada... yo lo pienso así, es para nada" Mujer, 51 años.

\section{Notas}

* Artículo de investigación. 